\title{
Correction: Targeted massively parallel sequencing of mature lymphoid neoplasms: assessment of empirical application and diagnostic utility in routine clinical practice
}

\author{
Adam R. Davis (1) - Sara L. Stone - Amanda R. Oran - Robyn T. Sussman - Siddharth Bhattacharyya • \\ Jennifer J. D. Morrissette $\cdot$ Adam Bagg
}

Published online: 22 December 2021

(c) The Author(s), under exclusive licence to Springer Nature America, Inc. part of Springer Nature 2021

Correction to: Modern Pathology

https://doi.org/10.1038/s41379-020-00720-7

The original version of this article unfortunately contained errors. All authors should only be affiliated with the Department of Pathology and Laboratory Medicine, Hospital of the University of Pennsylvania and Perelman School of Medicine at the University of Pennsylvania, Philadelphia, PA, USA. There also appears to be an error in the final layout of Fig. 3. Figure 3 should have its size increased and should be rotated to fill an entire page in landscape orientation for better resolution of the data. In the pdf print, it appears that the size of this figure was increased but that there was no change in orientation so that the Figure extends beyond the margins of the printed area. This eliminates vital information from the Figure compromising the value of the displayed data. The original article has been corrected. 